\title{
UNIFORM BOUNDS FOR THE COMPLEMENTARY INCOMPLETE GAMMA FUNCTION
}

\author{
JONATHAN M. BORWEIN AND O-YEAT CHAN
}

Abstract. We prove upper and lower bounds for the complementary incomplete gamma function $\Gamma(a, z)$ with complex parameters $a$ and $z$. Our bounds are refined within the circular hyperboloid of one sheet $\{(a, z):|z|>c|a-1|\}$ with $a$ real and $z$ complex. Our results show that within the hyperboloid, $|\Gamma(a, z)|$ is of order $|z|^{a-1} e^{-\operatorname{Re}(z)}$, and extends an upper estimate of Natalini and Palumbo to complex values of $z$.

Mathematics subject classification (2000): 33B20.

Keywords and phrases: Incomplete Gamma Function, Inequalities, Uniform Bounds.

\section{REFERENCES}

[1] H. AlzER, On some inequalities for the incomplete gamma function, Math. Comp. 66 (1997), no. 218, $771-778$.

[2] D. BorweIn, J. M. BorweIn, AND R. E. CRANDALl, Effective Laguerre asymptotics, Preprint available at http://locutus.cs.dal.ca:8088/archive/00000334/

[3] W. GAUTSCHI, The incomplete gamma functions since Tricomi, in Tricomi's ideas and contemporary applied mathematics (1997), 203-237.

[4] P. NATALINI AND B. PALUMBO, Inequalities for the incomplete gamma function, Math. Inequal. Appl. 3 (2000), no. 1, 69-77.

[5] R. B. PARIS, A uniform asymptotic expansion for the incomplete gamma function, J. Comput. Appl. Math. 148 (2002) no. 2, 323-339.

[6] F. QI, Monotonicity results and inequalities for the gamma and incomplete gamma functions, Math. Inequal. Appl. 5 (2002), no. 1, 61-67.

[7] N. M. TEMME, Uniform asymptotic expansions of the incomplete gamma functions and the incomplete beta function, Math. Comp. 29 (1975), no. 132, 1109-1114.

[8] N. M. TEMME, Uniform asymptotics for the incomplete gamma function starting from negative values of the parameters, Methods Appl. Anal. 3 (1996), no. 3, 335-344.

[9] F. TRICOMI, Asymptotische Eigenschaften der unvollständigen Gammafunktion, Math. Z. 53 (1950) $136-148$.

[10] G. N. WATson, A Trestise on the Theory of Bessel Functions, Cambridge University Press, Cambridge, 1922. 